\title{
Identification of damage and cracking behaviours based on energy dissipation mode analysis in a quasi-brittle material using Digital Image Correlation
}

\author{
Paul Leplay · Julien Réthoré · Sylvain Meille · \\ Marie-Christine Baietto
}

Submitted: February 2011

\begin{abstract}
Characterizing the crack stability to predict the behaviour of ceramics designed for industrial use is a challenging issue. It requires accurate crack tip detection during the controlled crack propagation of notched bending tests. Different indirect methods are available, like for instance the compliance technique. Recently, techniques based on Digital Image Correlation (DIC) have emerged: Finite-Element DIC (FE-DIC) with a finite element decomposition of the displacement field, Integrated-DIC (I-DIC) based on Williams'series decomposition of the displacement field and Regularized-DIC (R-DIC) for mechanical constraints. These full-field techniques enable the quantification of the crack length and the stress intensity factor $K^{I}$.

In this paper, these four methods are compared in terms of measurements of crack lengths and stress intensity factors during a notched bending test. The tested material is damageable quasi-brittle ceramic at room temperature. The non linearity of the stress-strain law of this microcraked ceramic results in a complex behaviour that is not captured by the compliance method during the bending test. Therefore the linear elastic compliance method leads to different estimation of crack lengths and stress intensity factors compared to DIC methods. On the other hand, the R-DIC approach handles the non linear material constitutive behaviour. It allows a deeper analysis of the mechanical fields, the energy dissipation and the damage mechanisms during the crack propagation.
\end{abstract}

Keywords Fracture - Damage · Digital Image Correlation · Equilibrium Gap Method · Quasi-Brittle Ceramic · Energy Dissipation · Bending Test

P. Leplay, J. Réthoré, M.-C. Baietto

Université de Lyon, CNRS INSA-Lyon, LaMCoS UMR 5259, F-69621 Villeurbanne, France

E-mail: julien.rethore@insa-lyon.fr

Tel.: +33-472438787 Fax.: +33-472438578

P. Leplay, S. Meille

Université de Lyon, CNRS INSA-Lyon, MATEIS UMR 5510, F-69621 Villeurbanne, France

P. Leplay

Saint-Gobain CREE, BP 224, F-84306 Cavaillon, France 


\section{Introduction}

Full-field optical methods are nowadays widely used in experimental mechanics thanks to the numerous existing techniques which enable sample visualization during mechanical tests. Different approaches like Moiré and Speckle Interferometry, Digital Image Correlation and Grid Methods [1,2,3] are used to capture and analyze such full-field data. These techniques can be used as displacement and strain measurement tools. However, they present a greater potential when associated with identification methods [4]. Indeed, displacement values during tests are rarely an end in themselves, contrary to quantities like mechanical properties which are key material characteristics. In fracture mechanics, one might be interested in measuring and quantifying quantities like the crack length, the stress intensity factors, the energy release rate, the crack propagation speed and the fracture process zone size.

Digital Image Correlation is now a well known full-field measurement technique. Based on the grey level conservation between two digital images of the specimen surface in two different states, this method enables accurate displacement quantification in 2D and 3D [5, $6,7,8]$. Recently, it has been proposed to decompose the displacement field over a set of finite element shape functions $[9,10]$. This procedure is particularly attractive because of the direct connection between experiments and simulations, since the kinematic bases may be identical in both cases. A large variety of specific basis functions may be used to integrate a chosen displacement decomposition into the measurement problem, like for beam kinematics [11,12,13] or fracture kinematics [14]. These approaches, called herein Integrated Digital Image Correlation (I-DIC), have been already associated with identification methods to quantify mechanical properties [15], establish damage laws [12] and determine stress intensity factors [16]. Unlike I-DIC approaches based on kinematics assumptions, it is also possible to a priori assume the mechanical behaviour of the material. It leads to a regularization of the obtained displacement field [17]. Theses approaches, called herein Regularized Digital Image Correlation (R-DIC), use the equilibrium gap method as a mechanical filter. According to the constitutive law of the tested material, all non admissible solutions are removed from the final displacement solution. These regularized approaches can also be used to identify mechanical quantities [18].

The purpose of this study is to characterize the crack growth resistance and the fracture toughness of a damageable structural ceramic. A notched four-point bending test was selected. This type of bending test is commonly used both in academic and industrial laboratories to characterize the crack resistance curve (R-curve). A R-curve shows the increase of resistance of a material, in terms of critical values of stress intensity factors with respect to crack lengths. This challenging issue requires an accurate crack detection as the load increases during the test. The elastic compliance method, usually employed to analyze this test, is briefly recalled in Section 2. Its underlying concepts are pointed out. Then, the DIC techniques are presented in Section 3. Section 3.4 is devoted to the analysis of the crack propagation during the four-point bending test according to these techniques. The results are compared and discussed. The regularized R-DIC method is presented in Section 4. The analysis of the notched bending test is then conducted according to the approach R-DIC. The differences between the results are commented and discussed. 


\section{Elastic Compliance Method}

The compliance method has been developed within the framework of Linear Elastic Fracture Mechanics [19]. Its main assumptions are an elastic behaviour of the material and tractionfree boundary conditions over the crack faces. The crack length $a_{i}$ is calculated at time $i$ by an iterative calculation based on the evolution of compliance:

$$
C_{i}=\frac{U_{i}}{P_{i}}, a_{i}=a_{i-1}+\left(\frac{W-a_{i-1}}{2}\right)\left(\frac{C_{i}-C_{i-1}}{C_{i}}\right)
$$

where $C_{i}$ is the sample compliance, $P_{i}$ the load, $U_{i}$ the deflection, $W$ the sample height. For most of the cases, the compliance must be calculated with respect to the 0-displacement 0-load point. Even if there is have important residual deflections for the cycling test (for instance Figure (2)), it may be the consequence of the presence of debris between crack faces which are not relevant for any stiffness considerations [20]. Using the crack length estimation given by equation (1), mode $I$ stress intensity factor $K^{I}$ is calculated for a notched four-point bending test according to elastic linear formulae [21]:

$$
\begin{aligned}
& K^{I}=\sigma_{i} \sqrt{\pi a_{i}} \chi\left(a_{i} / W\right), \sigma_{i}=\frac{3 P_{i}\left(D_{1}-D_{2}\right)}{2 B W^{2}} \\
& \chi\left(a_{i} / W\right)= \\
& \sqrt{\frac{2 W}{\pi a_{i}} \tan \left(\frac{\pi a_{i}}{2 W}\right)} \frac{0.923+0.199\left(1-\sin \left(\frac{\pi a_{i}}{2 W}\right)\right)^{4}}{\cos \left(\frac{\pi a_{i}}{2 W}\right)}
\end{aligned}
$$

where $B$ is the sample width, $D_{1}$ and $D_{2}$ the lower and upper spans.

Others fracture parameters can be calculated from load-displacement curves. It is possible to mention the methods developed by Rice et al. [22], Sakai et al. [23] and Garwood et al. [24]. For crack length estimation, all these methods are based on compliance considerations. But equation (2) implies systematically that a compliance variation is related to a unique damage mechanism, an incremental extension $d a$ of the main crack.

To determine the crack tip position on the specimen surface, direct optical observations are sometimes possible $[25,26]$ but it might be inaccurate and subjective especially if the surfaces can not be polished. The next section is devoted to alternative methods, such as full-field measurement techniques, to monitor continuously the crack extension during the test.

\section{Digital Image Correlation with kinematics Integration (I-DIC)}

3.1 General principle of DIC: optical flow conservation

DIC is a full field measurement method which enables one to capture both local and global variations of the displacement fields of a structure. Introduced in the 1980s [1,2], this method is now widely used to obtain quantitative data from various images.

Let us call $f$ the reference image, $g$ the deformed image, $u$ the displacement field and $b$ the noise. It is postulated that the texture of the sample surface is passively advected and its 
evolution between $f$ and $g$ is relevant from the structure displacement $u$ only. Thanks to this optical flow equation, the correlation method consists in comparing the two digital images ( $f$ and $g$ ) to evaluate the displacement field for each pixel of coordinate $x$ :

$$
f(x)=g(x+u(x))-b(x)
$$

Additional assumptions are considered to determine $v$, the best approximation possible of $u$ despite the noise $b$. Solving this inverse problem on a chosen region $\Omega$ may lead to the minimization of the global error thanks to a functional $\varphi_{c o r}^{2}$ :

$$
\begin{gathered}
v(x)=\operatorname{Arg} \operatorname{Min}\left(\varphi_{\text {cor }}^{2}\right) \\
\varphi_{\text {cor }}^{2}(x)=\iint_{\Omega}[f(x)-g(x+v(x))]^{2} d x
\end{gathered}
$$

The displacement field inside $\Omega$ is decomposed over a set of functions to regularize this ill-posed problem. $\psi_{n}$ are the basis functions and $\alpha_{n}$ the associated unknown degrees of freedom. The field $v$ is decomposed as follows:

$$
v(x)=\sum_{n} \alpha_{n} \psi_{n}(x)
$$

A non-linear least-squares algorithm leads to the resolution of a linear system:

$$
\begin{aligned}
& {\left[\int \int _ { \Omega } \left(\nabla g(x+v) \cdot \psi_{m}(x)\left(\nabla g(x+v) \cdot \psi_{n}(x) d x\right] d \alpha_{n}=\right.\right.} \\
& \iint_{\Omega}\left(f(x)-g(x+v)\left(\nabla g(x+v) \cdot \psi_{m}(x)\right)\right) d x
\end{aligned}
$$

where the summation over the repeated indices holds. Once convergence is achieved, the approximated displacement field $v$ is reconstructed on $\Omega$. The final correlation error $R_{c o r}$ is obtained by comparing the actual reference image $f$ and a corrected deformed image calculated from the deformed image $g$ and the displacement approximation $v$ :

$$
R_{\text {cor }}=|f(x)-g(x+v(x))|
$$

It indicates whether the displacement field satisfies locally the optical flow equation correctly or not.

\subsection{Finite-Element DIC or FE-FIC}

The displacement field decomposition in equation (7) is an essential step in DIC technique. A large variety of functions may be considered. The usual approaches of DIC consider constant or bilinear displacement forms inside independent regions of interest [2]. FiniteElement (FE) functions are particularly well suited $[9,10]$ and enable some uncertainty reductions with the same level of pixel data. In the following a DIC analysis carried out using FE shape functions will be denoted as FE-DIC.

However, such general decompositions do not allow for a direct extraction of searched mechanical quantities (Young's modulus, Poisson's ratio, damage law, stress intensity fac- 
tors...). If additional information is known concerning the form of the searched displacement, some global kinematic assumptions can be introduced through specific displacement basis functions $\psi_{n}$ : Roux et al. [14] used Williams' series around the tip of a crack for handling stress intensity factor measurements, Hild et al. [11] have employed a linear curvature element for accounting for cantilever beam kinematics, Leplay et al. [12] used specific functions for four-points bending and uniaxial tests kinematics. NURBS functions were also used by Réthoré et al. for handling high order beam kinematics [13]. Constructing the displacement basis functions usually allows having the searched mechanical quantities as direct unknowns of the DIC analysis (e.g., stress intensity factors, beam curvature, homogeneous strain field...). Further, compared to FE decomposition, the number of degrees of freedom involved is usually at least two orders of magnitude lower. Starting from a mesh with several thousands degrees of freedom with FE-DIC approach, it is possible for instance to have less than ten unknowns thanks to specific basis functions $[12,14]$. This allows for tremendous uncertainty reduction. However, one has to be careful and it is useful to check using $R_{\text {cor }}$ that the kinematic assumptions are fulfilled (Equation 9).

Section 3.3 presents a displacement decomposition dedicated to a crack kinematics [14, $27,16]$. The global kinematics is hence $a$ priori integrated into the measured displacement field problem. This technique is usually called Integrated-DIC (I-DIC).

\subsection{Integrated DIC or I-DIC}

Capturing the displacement discontinuity across the crack faces, enriched finite element kinematics have been employed in [28]. AS FE-DIC, this approach, based on X-FEM, requires the measurement of many degrees of freedom and might be very noise-sensitive in case of low strains. An other alternative is here used based on the use of the analytical displacement field expressions proposed by Williams [29] as performed by [14]. These expressions are applicable to a semi-infinite straight (along the $x_{1}$-axis) crack in an elastic isotropic infinite medium with traction-free boundary conditions over the crack faces. The displacement field solution is a double infinite complex serie, expressed in plane polar coordinates $\left(z=r e^{i \theta}\right.$ in the complex plane) with the origin centered at the crack tip. The mode $I$ and $I I$ solutions are expressed as follows:

$$
\left\{\begin{aligned}
\phi_{n}^{I}(z)=\phi_{n}^{I}(r, \theta)= & r^{n / 2}\left(\kappa e^{i n \theta / 2}-\frac{n}{2} e^{i(4-n) \theta / 2}\right. \\
& \left.+\left(\frac{n}{2}+(-1)^{n}\right) e^{-i n \theta / 2}\right) \\
\phi_{n}^{I I}(z)=\phi_{n}^{I I}(r, \theta) & =i r^{n / 2}\left(\kappa e^{i n \theta / 2}+\frac{n}{2} e^{i(4-n) \theta / 2}\right. \\
& \left.-\left(\frac{n}{2}-(-1)^{n}\right) e^{-i n \theta / 2}\right)
\end{aligned}\right.
$$

where $\kappa$ is the Kolossov's constant $(\kappa=(3-4 v)$ for plane strain or $\kappa=(3-v) /(1+v)$ for plane stress conditions, $v$ being the Poisson's ratio). All these fields are homogeneous functions of the distance to the crack tip $r$. All elastic displacement fields with finite strain energy density are generated with $0 \leq n<\infty$. All odd indices label fields having a discontinuity across the crack faces whereas even indices correspond to continuous fields. More 
precisely, $n=0$ order field corresponds to a translation parallel to the crack faces for mode $I$, orthogonal to the crack faces for mode $I I ; n=1$ order field gives access to the classical mode $I$ and mode $I I$ displacement fields which are proportional to stress intensity factors $K^{I}, K^{I I}$; $n=2$ order field corresponds to the T-stress component for mode $I$, and rigid body rotation about the crack tip for mode $I I$. Higher order fields account for the influence of non-fulfilled assumptions, like the influence of the boundary conditions (i.e. non-infinite medium). This set of fields is thus an appropriate basis functions to describe the displacement field for a traction free crack in an elastic medium.

$v(r, \theta)$ is thus rewritten considering this series as basis functions onto which the displacement field (7) is projected:

$$
v(z)=\sum_{n, j} \alpha_{n}^{j} \phi_{n}^{j}(z)
$$

where $\alpha_{n}^{j}$ are the unknown degrees of freedom i.e., the contribution of each field $\phi_{n}^{j}(r, \theta)$ is proportional to the amplitude $\alpha_{n}^{j}$. Associated with non-physical infinite energies at the crack tip when $r \rightarrow 0$, the subsingular $n<0$ fields have to be taken into account in order to verify the crack kinematics measurement quality [16]. Indeed, the influence of a wrong estimation of the equivalent crack tip location or the influence of the presence of a non negligible fracture process zone (FPZ), far away from the crack tip is quantified thanks to these $n<0$ fields. For instance, let us assume a mislocated crack tip with a distance $d$ along the $x_{1}$-axis. The polar frame being centered at the mislocated crack tip position, real crack tip coordinates are actually $(d, \theta=0)$.

$$
v(z+d)=\sum_{n, j} \widetilde{\alpha}_{n}^{j} \phi_{n}^{j}(z+d)
$$

where $\widetilde{\alpha}_{n}^{j}$ are the values of the degrees of freedom obtained for this crack mislocation. Using the recurrence formula (equation (13)) and a linearization, the equation (14) is obtained between the degrees of freedom with and without crack mislocation:

$$
\begin{gathered}
\frac{\partial \phi_{n}^{I}}{\partial x_{1}}=\frac{n}{2} \phi_{n-2}^{I} \\
\alpha_{n}^{I}=\widetilde{\alpha}_{n}^{I}-\frac{n+2}{2} d \widetilde{\alpha}_{n+2}^{I}
\end{gathered}
$$

Considering that $\alpha_{-1}^{I}$ must be zero to avoid diverging energy density, it is possible to show that a mislocation of the crack tip is responsible for a non-zero contribution of the subsingu$\operatorname{lar} \phi_{-1}^{I}$ field. Writing the equation 14 with order $n=-1$ and $j=I$, the distance $d$ is directly estimated from the ratio between $\widetilde{\alpha}_{1}^{I}$ and $\widetilde{\alpha}_{-1}^{I}$ :

$$
d=\frac{2 \widetilde{\alpha}_{-1}^{I}}{\widetilde{\alpha}_{1}^{I}}
$$

Thanks to this relationship, the offset $d$ between the estimated crack tip location and the actual one is quantified so that the polar frame origin can be shifted to the point $(d ; \theta=0)$. A new calculation with the new crack tip location is necessary to check whether $\alpha_{-1}^{1}$ is 
actually nil or not. The minimization of the contribution of the supersingular field $\phi_{-1}^{I}$ using an iterative algorithm yields to an accurate positioning of the equivalent elastic crack tip in the image along the axis $\theta=0$ [14].

Note that the very small region surrounding the crack tip must be excluded from the region $\Omega$ for two reasons. First, one knows that the elastic fields (Equation (10)) can not describe all the non linear phenomena which classically occur at the crack tip (plasticity, damage...). Then, the supersingular fields (like $\phi_{-1}^{I}$ ) are singular when $r \rightarrow 0$. Therefore a mask is used to exclude from the calculation the region in the immediate vicinity of the crack. The crack mask radius must be as small as possible to enable the supersingular fields to detect correctly a crack tip mislocation [30].

It also means that all the quantitative information about the crack behaviour brought by this I-DIC formulation do not come from the crack itself but from the fields which irradiate away from the crack projected on an elastic basis.

\subsection{Application to notched bending tests}

\subsubsection{Experimental Procedure}

Aluminium titanate $\mathrm{Al}_{2} \mathrm{TiO}_{5}$ is a micro cracked ceramic due to the anisotropic thermal expansion coefficient of its crystals [31]. The microcracks appear at grain boundaries during cooling from the sintering temperature. Because of these microcracks, this quasi-brittle ceramic strengthened with a secondary silicate phase becomes an excellent potential material for industrial thermostructural applications. The influence of the sintering process, the microstructure, the secondary silicate phase and the thermal expansion coefficient has been widely studied [32,33] but mechanical and fracture aspects are rare in literature $[34,35,36]$. However, this ceramic is known to be damageable at room temperature because of the microcrack propagation [12]. It is however essential in a design stage to have access to crucial property such as the fracture toughness and to the knowledge of the crack resistance curve, particularly if it exhibits an increase of resistance with increasing crack length.

The tested material is mainly made of aluminium titanate with a secondary silicate phase. It exhibits a high porosity volume fraction varying from 40 to $50 \%$. The samples were extruded then sintered for a final rectangular section of $\mathrm{B} \times \mathrm{W}=5.3 \times 7.0 \mathrm{~mm}^{2}$. The notches, made with a steel blade, were $1 \mathrm{~mm}$ long $\left(\alpha=a_{0} / W=0.14\right)$ and $180 \mu \mathrm{m}$ thick. Four-point bending tests were performed on a hydraulic machine (Instron 8502) with a $5000 \mathrm{~N}$ load cell. The lower span was $D_{1}=60 \mathrm{~mm}$ and the upper span $D_{2}=20 \mathrm{~mm}$. A linear variable differential transducer (LVDT) was used to control the deflection at the middle of the beam at a rate of $100 \mu \mathrm{m} / \mathrm{min}$.

A CCD camera was used to continuously record images of the sample surface during each mechanical test. The maximum image resolution of this camera is $1200 \times 1600$ pixels with an 8-bit digitization for grey levels. The acquisition frequency was one image every second. The optical magnification of classical lenses depending on the distance to the sample surface, out-of-plane displacements or misalignments might induce artificial magnifications interpreted as in-plane strains by the DIC system. To overcome this possible error, the CCD camera was fitted with a $200 \mathrm{~mm}$ telecentric lens, ensuring a magnification independent on 
the distance. Each pixel on the captured images has here a $10.9 \mu \mathrm{m}$ physical size.

Five notched samples were tested. The averaged maximum load is $14.8 \pm 0.4 \mathrm{~N}$. The behaviour is non linear with a stable crack propagation (Figure 3). Monotonous and cycling tests lead to similar envelope curves. One must note that the global response of this quasibrittle porous ceramic is highly repetitive on these mechanical tests. From a material point of view, this quasi-brittleness is the consequence of the growing microcracks creating a non negligible fracture process zone around the crack tip. Using the BSE mode (Bask-Scattered Electrons) for a better vizualisation, SEM pictures reveal the presence of multicracking, branching and debonding (Figure 1 and 2). All the phenomena are energy-consuming. The important residual deflection for the cycling test illustrated in Figure 3 can be associated to friction phenomena between microcrack faces and the presence of fragments between the main crack faces. Therefore the sample compliance is better estimated with respect to the 0-displacement 0-load point [20,37].

\subsubsection{Different analyzing methods}

The compliance method based on the load-deflection curve briefly recalled in section 2, FEDIC and I-DIC approaches presented in section 3 are used to analyze the four-point bending test described above. Concerning FE-DIC, square finite elements were chosen. This leads to the choice of Q4 finite elements as the simplest basis. The calculation was performed on a regular mesh made of 20x41 quadrangular elements (element size $=32$ pixels). With this FE-DIC approach, 1764 degrees of freedom have thus to be measured. A global region of interest is considered as shown in Figure 4. As the bi-linear basis functions assume a continuous displacement, the correlation residual $R_{\text {cor }}$ might rise for regions where this assumption is not fulfilled. Concerning I-DIC analysis, the global region of interest is also shown in figures 1 and 4. Mode $I$ and mode $I I$ field orders from $n=-3$ to $n=+7$ are considered since they are sufficient to capture faithfully the crack kinematics [38]. Indeed, larger negative order fields vanish very quickly with $r$ and can be omitted from the analysis. Similarly, large positive indices do not affect the crack tip kinematics and are only useful for accounting for very inhomogeneous boundary conditions [16]. It has been checked that considering fields orders from $n=-5$ to $n=+9$ does not change the solution but only slow down the algorithm convergence. With the I-DIC approach based on these fields, only 22 degrees of freedom have thus to be measured. Since the crack is not perfectly straight, the mask radius is set to 20 pixels in our case. It has been verified that using a mask with a radius between 15 and 40 pixels does not affect the solution accuracy.

The location of the equivalent elastic crack tip is estimated through the minimization of $\alpha_{-1}^{I}$. The stress intensity factor values are directly obtained from the measured amplitudes of $\alpha_{1}^{I, I I}$.

\subsubsection{Results and comparison}

The axial displacement map obtained using FE-DIC and I-DIC approaches are presented in Figure 5. FE-DIC displacement map is quite noisy since it is based on numerous degrees of freedom. It is still possible to identify the crack geometry using the correlation residual 
$R_{\text {cor }}$. The averaged correlation residual is low $(0.49 \%)$ but the map indicates a steep localization corresponding to the place where the displacement continuity assumption fails. The prediction of the crack path is thus better than those performed according to the axial displacement, but the crack tip location is rough, inaccurate and subjective. The displacement field is further not sufficiently smooth to be derived to obtain the strain fields.

On the other hand, I-DIC displacement map is perfectly smooth since the displacement field is based on few analytical basis functions. The homogeneous and low correlation residual $(0.47 \%$ in average over the region of interest excluding the mask aroung the crack) indicates that the basis functions detailled in Section 3.3 allow capturing the displacement at the sample surface within the region that has not been masked. Besides, no post-processing is required since the crack tip location and the stress intensity factor are directly obtained from the degrees of freedom.

The crack length versus the vertical deflection is plotted on Figure 6 according to the compliance, FE-DIC (manual tracking on the correlation error map) and I-DIC methods. The calculations have been stopped for a maximum vertical deflection of $130 \mu \mathrm{m}$. For greater defections, the crack tip is too close from the rear compressive part of the beam and is submitted to boundary effects that cannot be accounted for by the displacement fields in Equation (10).

Figure 6 shows differences between the estimated crack lengths versus the method employed for their estimation. According to the compliance method, a compliance variation is related to a unique damage mechanism, an incremental crack extension $d a$. The initiation and propagation of a microcrack network within a process zone around the crack tip or diffuse damage that weakens the sample can be interpreted only as a growth of the main elastic crack. Therefore, this method overestimates the crack length for small loads (during the elaboration of the process zone) before underestimating it for greater loads. Concerning, FE and I-DIC methods, the estimated crack lengths are very close. This confirms that the compliance leads in the present case to an wrong estimation of the crack length due to its underlying assumptions. However, it is not possible from I-DIC results to identify the mechanism responsible for the earliest damage of the sample. The measurement of the crack tip at the specimen surface with DIC approaches involves the problem of the crack front curvature over the specimen thickness. This problem has already been studied for non-brittle ceramics [25,39]. For some rectangular samples, as thick as ours, no remarkable crack curvature was found and the maximum error in crack length measurement was estimated to be $\pm 2 \%$ at the crack surface. Indeed, no plastic zone and crack tunnelling exist for ceramics, as it is found in metallic materials in cases of plain stress and plain strain. Therefore, the difference observed between compliance and DIC methods on this porous ceramic might not come from thickness effects but from the damage caused by the microcracks.

Concerning the SIF variations, FE-DIC results were not computed, as mentioned previously (Section 3.2). A great difference between compliance and I-DIC stress intensity factor values is highlighted by Figure 7. This figure indicates that for similar vertical deflection, $K^{I}$ values according to the two methods are different, and that the gap increases with the increasing deflection. Again, as the compliance assumptions are violated, any energy dissipation is seen by the compliance method as a propagation of the main crack. Our interpretation is the following. Any loss of global stiffness leads to crack extension following the 
compliance method. Further, as the load is lower than the one that should have been applied for the same crack within an elastic material, the estimated SIF is much lower than the one computed from I-DIC. Indeed, I-DIC leads to a kinematic-based estimate of $K^{I}$. The displacement field accounting for the loss of linearity of the material leads to a larger $K^{I}$ value than the one computed using the compliance method. A ratio of about 2 is reported between both $K^{I}$ compliance and I-DIC factors.

In Section 4, we propose to analyze this test using a original DIC technique that accounts for the non-linear constitutive law of the material. The aim is to distinguish the respective influence of diffuse and localized damages in the crack tip vicinity. Further, an energy analysis is proposed and the amount of energy dissipated by each damage mechanism is evaluated.

\section{Digital Image Correlation with mechanical Regularization (R-DIC)}

In the previous section 3, global correlation techniques have been implemented. The displacement decomposition rests either on continuous finite element shape functions or Williams' series functions for a cracked elastic medium. Information on the material constitutive law and momentum equation can also be added into the displacement field functions. Section 4 presents this approach based on the equilibrium gap method [17]. The displacement solution is mechanically regularized with respect to the material constitutive law. Let us call this technique Regularized-DIC (R-DIC).

4.1 The equilibrium gap method as a mechanical filter

The displacement field decomposition over a set of FE functions provides a suitable tool for coupling measurements and numerical simulations. Indeed, the discretization schemes are identical for both. In case of very small displacements and strains (noisy measurements), it is however unrealistic to compute stress fields directly from displacements. The R-DIC basic concept is to add the equilibrium state constraint directly into the displacement identification procedure to "mechanically" filter the unbalanced components of the displacement field.

The momentum equation written in a discretized formalism using FE shape functions, states that the internal force vector equals the external force vector:

$$
\left\{F_{\text {int }}\right\}=\left\{F_{\text {ext }}\right\}
$$

In the absence of body force, the external force vector $\left\{F_{\text {ext }}\right\}$ has non-zero value only at the nodes where boundary conditions (prescribed displacement or non-zero stress/force) are applied. For the analyzed sample, these nodes are those on the left and right edges of the mesh. Let us denote by $\left\{\bar{F}_{\text {int }}\right\}$ the restriction of the internal force vector to nodes where $\left\{F_{\text {ext }}\right\}$ vanishes. It is proposed to add to the minimization of $\varphi_{c o r}^{2}$ an equilibrium gap condition $\varphi_{m e c}^{2}$ :

$$
\varphi_{m e c}^{2}=\frac{1}{2}\left\{\bar{F}_{\text {int }}\right\}^{T}\left\{\bar{F}_{\text {int }}\right\}
$$


as a penalty term with $\lambda$ the penalty parameter:

$$
\varphi_{\text {tot }}^{2}=\varphi_{c o r}^{2}+\left(\frac{1}{\lambda}-1\right) \varphi_{m e c}^{2}
$$

The algorithm is aimed at minimizing at best the correlation residual of equation (9) with a penalization of the equilibrium gap equation (17). All the solutions not mechanically balanced are canceled by $\varphi_{m e c}^{2}$, so that the final displacement field is regularized with respect to the given constitutive law. The penalty parameter $\lambda$ belongs to $[0 ; 1]$. If $\lambda=1$, the mechanical residual has no influence and the algorithm behaves like usual correlation algorithm used in FE-DIC. If $\lambda \rightarrow 0^{+}$, the algorithm behaves more less like a Dirichlet finite-element calculation with displacement boundary conditions coming from DIC (the mechanical residual is defined only for the internal nodes, the elements at the mesh boundary are always solved according to DIC only without mechanical regularization). It has to be pointed out that the penalty parameter $\lambda$ introduces a length scale below which mechanical considerations dominate and above which DIC dominate [17]. Therefore this regularizing approach acts as a frequency low-pass filter, removing all wavelengths which are not mechanically admissible.

As R-DIC allows measuring a displacement field fulfilling at best the momentum balance for a given a constitutive law, it is possible to compute the corresponding stress field and to evaluate how the damage spreads within the sample. This is now illustrated by the analysis of the notched bending tests previously studied in Section 3.4.

\subsection{Application to notched bending tests}

R-DIC is now applied to the same notched bending tests previously analyzed using the compliance method, FE and I-DIC in Section 3. Figure 8 shows the global area of interest considered for R-DIC. An unstructured mesh made of T3 elements, conforming to the geometry of the notch, is used. The averaged size of these triangle elements is about 10 pixels. The region covered by this unstructured mesh is identical to the one considered in Section 3. It is necessary to use a constitutive law adapted to the tested material. At room temperature, the stress-strain ceramic constitutive and damage laws were identified by the authors in a previous paper [12] using a specific DIC approach. Unnotched bending tests were analyzed using DIC and specific basis functions dedicated to bending kinematics were employed for the displacement decomposition. A dissymetric behaviour between tension and compression was identified. The ceramic behaviour is elastic linear in compression whereas it is damageable in tension because of microcrack propagation. In tension, a Chaboche-Lemaitre damage model is used [40]. The damage evolution is expressed as a function of an equivalent strain $\varepsilon_{e q}:$

$$
D\left(\varepsilon_{e q}\right)=a \cdot\left(1-\exp \left(-\hat{\varepsilon}_{e q} / b\right)\right)
$$

where $\hat{\varepsilon}_{e q}$ denotes the maximum value over time of $\varepsilon_{e q}, a$ and $b$ are material parameters previously identified in [12]: $a=0.886, b=0.00141$ and $D_{\max }=0.71$. The value $D_{\max }$ corresponds to the maximum damage that the material can support before unstable failure occurs. A 
Mazars equivalent strain [41] is used herein:

$$
\varepsilon_{e q}=\left\langle\varepsilon_{1}\right\rangle_{+}+\left\langle\varepsilon_{2}\right\rangle_{+}+\frac{v}{1-v}\left\langle-\varepsilon_{1}-\varepsilon_{2}\right\rangle_{+}
$$

where $\varepsilon_{1,2}$ are the principal strains and $\langle\bullet\rangle_{+}$the Macaulay brackets (i.e., the positive part of the inner argument). When $D=D_{\max }$ within an element, failure occurs and the element stiffness is enforced to zero by setting $D=1$.

Réthoré [18] shows that results from R-DIC are almost independent on the value of the penalty parameter $\lambda$. In the present case, additional difficulties arise concerning the choice of this parameter. It has been mentioned above that $\lambda$ introduces a cut-off frequency of a low-pass filter. For very low value of $\lambda$ (e.g., $10^{-6}$ ), the cut-off frequency is as large as R-DIC behaves more or less as a Dirichlet FE simulation. As the signal / noise ratio of pure DIC analysis is really low in the present analysis on a ceramic, the "boundary conditions" are so noisy that the elements on the boundary are damaged and broken (from the given failure criterion) at the very first step of the analysis. The penalty parameter has thus to be chosen carefully. It is proposed to use the stress field from R-DIC as data to determine over a vertical beam section the global load by integrating the moment with respect to the neutral axis location $n$ :

$$
P_{R-D I C}=\frac{4 B}{D_{1}-D_{2}} \int_{-W / 2}^{+W / 2} \sigma(x) \cdot(x-n) d x
$$

This computed load $P_{R-D I C}$ is then compared to the experimental recorded one in order to calibrate the appropriate penalty parameter value $\lambda$. A very good agreement between the experimental and the computed values of the load is observed for $\lambda$ varying roughly between $1.10^{-2}$ and $1.10^{-1}$. Within this interval, the measured kinematics corresponds to an equilibrium state relevant for the crack problem. This validity interval, smaller than those obtained in the literature $[17,18]$, is a consequence of the low ratio between data and noise due to the small level of strains of ceramics. Compared to the literature, it is also the first time that this approach is applied to a damageable material. It brings additional complexity and makes the identification problem more ill-posed. Some developments are possible to improve the quality of the strain measurement and damage calculation at the borders. It might increase the independance of the R-DIC solution with respect to the parameter $\lambda$ in our case, this point should be tackled in a future paper.

Stress intensity factors and crack tip position are extracted from R-DIC displacement by using a least-square minimization of the gap between $v_{R-D I C}$ and a decomposition over Williams' series. The corresponding coefficients are obtained by minimizing:

$$
\alpha_{n}^{j}=\operatorname{Arg} \operatorname{Min}\left(\iint_{\Omega}\left[v_{R-D I C}(\lambda, x)-\sum_{n, j} \alpha_{n}^{j} \phi_{n}^{j}(x)\right]^{2} d x\right)
$$

In the following, $\lambda$ is set to $4.10^{-2}, n$ ranges from -3 to 7 and $j$ considers mode $I$ and mode II fields. The results are then compared to those obtained using I-DIC and compliance methods (Section 3.4). Figure 11 shows the crack length evolution. One notices that R-DIC gives results similar to the ones obtained according to I-DIC meaning that the identified crack advance kinematics was preserved by the mechanical filter. Previously, a possible diffuse damage was invoked to explain the differences between the crack length evolution 
obtained from I-DIC (and now R-DIC) and compliance methods. R-DIC gives access to the damage distribution over the entire specimen surface. Figure 9 shows the axial displacement fields and damage fields obtained using R-DIC for some specific load steps, corresponding to load steps labeled (a), (b), (c) and (d) on Figure 6. One observes the main crack propagation but also the diffuse damage spreading over the entire specimen. Note the influence of the nodes on the left and right edges that do not hold mechanical regularization and induce a short scale perturbation in the mechanical fields. For Figure (9a) and Figure (9d), the damage field is plotted at the step before the crack is detected from both I-DIC and R-DIC displacement fields. It shows that at non-zero damage value is obtained over a region that is not restricted to the vicinity of the crack tip. This leads to a significant reduction of the global stiffness of the specimen that is interpreted as the propagation of the main crack for the compliance method.

During the next steps of the test, the damage concentrates around the crack tip as shown by Figure 9. It is consistent with SEM pictures showing that branching and debonding phenomena occur in the vicinity of the crack tip (Figure 2). Further, if one compares the value of $K^{I}$ computed from the three analyses, there is again a very good agreement between I-DIC and R-DIC whereas values from the compliance method are about half of the values from both DIC analyses, as mentioned earlier.

It is thus clear that there is a competition between diffuse damage and localized damage (i.e., crack propagation).

\subsection{Energy based analysis}

To get an improved understanding of this test and of the damage behaviour of this ceramic, it is proposed to quantify the energies dissipated by the different damage modes. The total cumulated energy dissipated during the experiment is evaluated from the load displacement curve [25]. Assuming an elastic unloading, the total cumulated dissipated energy $W_{t o t}$ is given by:

$$
W_{t o t}=\int_{0}^{u_{e x p}(t)} P_{\exp } \mathrm{d} u_{\exp }-\frac{1}{2} P_{\exp }(t) u_{\exp }(t)
$$

It is assumed that this total dissipated energy can be partitioned into a fracture energy due to new free surface creation $W_{f}$ and into a diffuse damage dissipation $W_{d}$ :

- The energy dissipated by diffuse damage can be evaluated by integrating the fields obtained by the R-DIC approach over the finite element mesh. It is equal to the difference between effective and real strain energies [42] :

$$
W_{d}=\frac{1}{2} \int_{\Omega} \sigma_{e}: \varepsilon_{r d i c} \mathrm{~d} \Omega-\int_{0}^{t} \int_{\Omega} \sigma_{r d i c}: \dot{\varepsilon}_{r d i c} \mathrm{~d} \Omega \mathrm{d} t
$$

where $\sigma_{e}$ are the effective stresses, associated to the strain field if the material was elastic, and $\sigma_{\text {rdic }}$ the real stresses, calculated thanks to the damageable constitutive law.

- Using the Linear Elastic Fracture Mechanics theory, the cumulated energy $W_{f}$ dissipated by the fracture process is the cumulated energy dissipated by the fracture process is related 
to the energy release rate as:

$$
W_{f}(t)=\int_{0}^{a(t)} G(t) \mathrm{d} a
$$

where $G$ is the energy release rate. $G$ can usually be related to stress intensity factor through the Irwin formula. However, Bui [19] pointed out that in the presence of non-linear (or transient) processes one must distinguish between a kinematic stress intensity factor $K_{u}^{I}$ related to the displacement "singularity" and a static stress intensity factor $K_{\sigma}^{I}$ related to the singularity of the stress field. I-DIC and the least-square minimization of the displacement gap for R-DIC lead to kinematic stress intensity factor $K_{u}^{I}$ evaluations as they are based on displacement field data. In order to estimate $K_{\sigma}^{I}$, R-DIC stress fields are analyzed and a stress gap between the computed field and the one derived from Williams' series assuming a linear elastic constitutive law, is minimized in a least square sense (Figure 12). The energy release rate is then evaluated in plane stress by:

$$
G=\frac{1}{E}\left(K_{u}^{I} K_{\sigma}^{I}+K_{u}^{I I} K_{\sigma}^{I I}\right)
$$

On Figure 13, we plot the evolution of these energies $W_{t o t}, W_{f}$ and $W_{d}$ as functions of the crack length obtained according to I-DIC. At the end, the total dissipated energy $W_{\text {tot }}$ is equal to $4,71 \cdot 10^{-4} \mathrm{~J}$, whereas the sum of $W_{f}$ and $W_{d}$ is equal to $4,61.10^{-4} \mathrm{~J}$. This difference being less than $3 \%$, the energy balance is validated. One observes that a small, but non-zero, amount of energy $\left(W_{d}=1,0.10^{-5} \mathrm{~J}\right)$ is dissipated by diffuse damage before crack initiation. Then the dominant dissipation mode is the localized mode (i.e., fracture). When the crack length becomes longer than $1.2 \mathrm{~mm}$, the dissipation by diffuse damage increases and reaches $30 \%$ of the total dissipated energy. At this transition length, the evolution of the damage field corresponds to a finite size effect. Indeed, from Figure (9d), it is observed that the free surface in front of the crack tip has an increasing influence on the stress field and that damage starts to develop from this free surface.

R-curves are plotted for R-DIC and compliance methods on Figure 14. It is obvious that the compliance method underestimates the resistance of the material as all non-linear processes are supposed to be solely related to crack propagation by this method. The deeper analysis proposed in this paper shows that the crack growth resistance of the material properly extracted from the experimental data is much higher (a factor of about 2 is obtained for the value of $G$ ). This result may impact directly the design of industrial applications of such quasi-brittle material.

\section{Conclusion}

The aim of this paper is to characterize the crack growth behaviour of a quasi-brittle ceramic and to quantify its crack growth resistance. To reach these goals, the elastic compliance method is usually used to analyze a notched bending test. In this paper, DIC techniques, which allow a meaningful crack detection through displacement field measurements, are used. Integrated-DIC method is based here on the displacement field decomposition over a set of shape functions adapted to the test kinematics, i.e., the Williams' series. Its allows a quantification of the displacement field with lower uncertainty levels. Besides, stress inten- 
sity factors and crack tip location are estimated without requiring post-processing. These results ( $K_{I}$ and crack tip position) are seen to be different from those obtained by the compliance method.

The crack tip positions with the specific approach I-DIC are obtained more directly and accurately than with the general approach FE-DIC based on finite element functions. For the compliance method, any energy dissipation is interpreted as a propagation of the main crack. Therefore it is suggested that the wrong crack initiation detected by the compliance method at the first loading steps is a consequence of non-linear processes.

To handle the non-linear mechanical behaviour, a Regularized-DIC formulation is adopted. This approach combinates the numerical and experimental aspects. It allows selecting a DIC displacement field which satisfies additional constraints coming from a penalization by the equilibrium gap with respect to a given constitutive law. The used law relies on a Chaboche-Lemaître damage model. By coupling energy dissipation analyses and R-DIC field projections onto Williams' series, it is possible to explain the differences obtained between the compliance and DIC methods. Further, it is possible to distinguish diffuse and local damages during the test. Interpreted in term of dissipated energy, three different stages appear. First, diffuse damage around the initial notch is shown to be the dominant damage mechanism responsible for the macro-crack initiation. Then, the macro-crack propagation starts and is detected by the I-DIC method and optical observations. The fracture mechanism dominates during this second phase. Last, finite size and boundary effects finally arise once the crack exceeds a certain length. The energy dissipating mode switches to diffuse damage close to the top edge of the sample.

Compared to standard DIC, the R-DIC approach allows accessing to a refined dechanical description of the damage phenomena during the test. The quantification of the energy dissipated allows distinguishing the diffuse and local mechanisms. An estimation of the crack growth resistance of the material is obtained without accounting for the bias inherent to the usual compliance method. It is concluded from these results, that the actual propagation law or resistance curve for the studied material is given by the R-DIC analysis.

Different improvements are possible in our case. First, the independance of the RDIC approach with respect to the penalty parameter must be increased thanks to a better measurement of the displacement and damage fields at the borders. Although the mechanical response of this porous quasi-brittle material is very repetitive, this analysis based on I-DIC and R-DIC approaches must be applied on others mechanical tests with different sample sizes to assure the validity of the identified damage and cracking behaviours.

We showed how an appropriate combination of measurement and simulation tools allows a deeper understanding and interpretation of non-linear fracture phenomena in a quasibrittle ceramic.

\section{Acknowledgments}

This work is financially supported by the ANRT (National Association for Research and Technology) through the contract $n^{\circ} 2008 / 501$. The authors wish to thank Fabiano Rodrigues 
and his colleagues at the CREE (Saint-Gobain group) for providing the samples and supporting this research project.

\section{References}

1. WH Peters and WF Ranson. Digital imaging techniques in experimental stress analysis. Optical Engineering, 21(3):427-431, 1982.

2. M.A. Sutton, W.J. Wolters, W.H. Peters, W.F. Ranson, and S.R. McNeill. Determination of displacements using an improved digital correlation method. Image Vision Comput, 1(3):133-139, 1983.

3. Y. Surrel. Full-field optical methods for mechanical engineering: essential concepts to find ones way. Composite Testing and Model Identification, 1:21-23, 2004.

4. S. Avril, M. Bonnet, A.S. Bretelle, M. Grediac, F. Hild, P. Ienny, F. Latourte, D. Lemosse, S. Pagano, E. Pagnacco, et al. Overview of identification methods of mechanical parameters based on full-field measurements. Experimental Mechanics, 48(4):381-402, 2008.

5. M. Sutton, S. McNeill, J. Helm, and Y. Chao. Advances in two-dimensional and three-dimensional computer vision. Photomechanics, 77:323-372, 2000.

6. Y.Q. Wang, M.A. Sutton, H.A. Bruck, and H.W. Schreier. Quantitative Error Assessment in Pattern Matching: Effects of Intensity Pattern Noise, Interpolation, Strain and Image Contrast on Motion Measurements. Strain, 45(2):160-178, 2009.

7. L. Robert, F. Nazaret, T. Cutard, and J.J. Orteu. Use of 3-d digital image correlation to characterize the mechanical behavior of a fiber reinforced refractory castable. Experimental Mechanics, 47(6):761-773, 2007.

8. J. Rannou, N. Limodin, J. Réthoré, A. Gravouil, W. Ludwig, M.-C. Baietto-Dubourg, J.-Y. Buffiere, A. Combescure, F. Hild, and S. Roux. Three dimensional experimental and numerical multiscale analysis of a fatigue crack. Computer Methods in Applied Mechanics and Engineering, 199:1307-1325, 2010.

9. G. Besnard, F. Hild, and S. Roux. Finite-element displacement fields analysis from digital images: application to portevin-le châtelier bands. Experimental Mechanics, 46(6):789-803, 2006.

10. Y. Sun, J.H.L. Pang, C.K. Wong, and F. Su. Finite element formulation for a digital image correlation method. Applied Optics, 44(34):7357-7363, 2005.

11. F. Hild, S. Roux, R. Gras, N. Guerrero, M.E. Marante, and J. Florez-Lopez. Displacement measurement technique for beam kinematics. Optics and Lasers in Engineering, 47(3-4):495-503, 2009.

12. P. Leplay, J. Réthoré, S. Meille, and M.-C. Baietto. Damage law identification of a quasi-brittle ceramic from a bending test using digital image correlation. Journal of the European Ceramic Society, 30:27152725, 2010.

13. J. Réthoré, T. Elguedj, P. Simon, and M. Coret. On the use of nurbs functions for displacement derivatives measurement by digital image correlation. Experimental Mechanics, 50(7):1099-1116, 2009.

14. S. Roux and F. Hild. Stress intensity factor measurements from digital image correlation: post-processing and integrated approaches. International Journal of Fracture, 140(1):141-157, 2006.

15. F. Hild and S. Roux. Digital image correlation: from displacement measurement to identification of elastic properties-a review. Strain, 42(2):69-80, 2006.

16. S. Roux, J. Réthoré, and F. Hild. Digital image correlation and fracture: an advanced technique for estimating stress intensity factors of 2d and 3d cracks. Journal of Physics D: Applied Physics, 42:214004, 2009.

17. J. Réthoré, S. Roux, and F. Hild. An extended and integrated digital image correlation technique applied to the analysis of fractured samples. European Journal Computational Mechanics, 18:285-306, 2009.

18. J. Réthoré. Optimal identification of mechanical properties from digital images. International Journal for Numerical Methods in Engineering, submitted, 2009.

19. H.D. Bui and P. Germain. Mécanique de la rupture fragile. Masson Paris, 1978.

20. M. Bouquet, JM Birbis, and JM Quenisset. Toughness assessment of ceramic matrix composites. Composites Science and Technology, 37(1-3):223-248, 1990.

21. H. Tada, P.C. Paris, and G.R. Irwin. The stress analysis of cracks handbook. Paris Productions Incorporated, 1985. 
22. J.R. Rice, P.C. Paris, and J.G. Merkle. Progress in flaw growth and fracture toughness testing. Journal of the American Ceramic Society, 536:231-245, 1973.

23. M. Sakai, K. Urashima, and M. Inagaki. Energy principle of elastic-plastic fracture and its application to the fracture mechanics of a polycrystalline graphite. Journal of the American Ceramic Society, 66(12):868-874, 1983.

24. S.J. Garwood, J.N. Robinson, and C.E. Turner. The measurement of crack growth resistance curves (r-curves) using the $\mathrm{j}$ integral. International Journal of Fracture, 11(3):528-530, 1975.

25. A. Bornhauser, K. Kromp, and RF Pabst. R-curve evaluation with ceramic materials at elevated temperatures by an energy approach using direct observation and compliance calculation of the crack length. Journal of Materials Science, 20(7):2586-2596, 1985.

26. J. Chevalier. Etude de la propagation des fissures dans une zircone 3Y-TZP pour applications biomedicales. PhD thesis, INSA de Lyon, 1996.

27. R. Hamam, F. Hild, and S. Roux. Stress intensity factor gauging by digital image correlation: Application in cyclic fatigue. Strain, 43(3):181-192, 2007.

28. J. Réthoré, F. Hild, and S. Roux. Extended digital image correlation with crack shape optimization. International Journal for Numerical Methods in Engineering, 73:248-272, 2008.

29. M.L. Williams. On the stress distribution at the base of a stationary crack. ASME Journal of Applied Mechanics, 24:109-114, 1957.

30. J. Rethore, S. Roux, and F. Hild. Optimal and noise-robust extraction of fracture mechanics parameters from kinematic measurements. Engineering Fracture Mechanics, 78(9):1827-1845, 2011.

31. H.A.J. Thomas and R. Stevens. Aluminium titanate: a literature review. i: Microcracking phenomena. British ceramic. Transactions and journal, 88(4):144-151, 1989.

32. I.J. Kim and L.J. Gauckler. Excellent thermal shock resistant materials with low thermal expansion coefficients. Journal of Ceramic Processing Research, 9(3):240-245, 2008.

33. A. Tsetsekou. A comparison study of tialite ceramics doped with various oxide materials and tialitemullite composites: microstructural, thermal and mechanical properties. Journal of the European Ceramic Society, 25(4):335-348, 2005.

34. C.H. Chen and H. Awaji. Temperature dependence of mechanical properties of aluminum titanate ceramics. Journal of the European Ceramic Society, 27(1):13-18, 2007.

35. K. Hamano, Y. Ohya, and Z. Nakagawa. Crack propagation resistance of aluminium titanate ceramics. International Journal of High Technology Ceramics, 1(2):129-137, 1985.

36. J.J. Melendez-Martinez, M. Jimenez-Melendo, A. Dominguez-Rodriguez, and G. Wotting. High temperature mechanical behavior of aluminium titanate-mullite composites. Journal of the European Ceramic Society, 21(1):63-70, 2001.

37. B.R. Lawn and D.B. Marshall. Nonlinear stress-strain curves for solids containing closed cracks with friction. Journal of the Mechanics and Physics of Solids, 46(1):85-113, 1998.

38. C. Henninger, S. Roux, and F. Hild. Enriched kinematic fields of cracked structures. International Journal of Solids and Structures, 1, 2010.

39. H. Weininger and R.F. Pasbst. Crack resistance behavior of ceramic materials. In -, page 84. 4th European Conference on Fracture, 1982.

40. J. Lemaître and J.L. Chaboche. Mecanique des Materiaux Solides (2nd ed.). Dunod, Paris, 1988.

41. J. Mazars. A description of micro-and macroscale damage of concrete structures. Engineering Fracture Mechanics, 25(5-6):729-737, 1986.

42. F. Cazes. Construction et implémentation de lois cohésives extrinsèques. PhD thesis, INSA de Lyon, 2010 . 


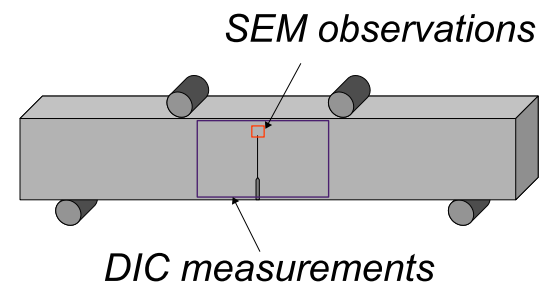

Fig. 1 Scheme of the bending test and zones for MEB observations and DIC measurements

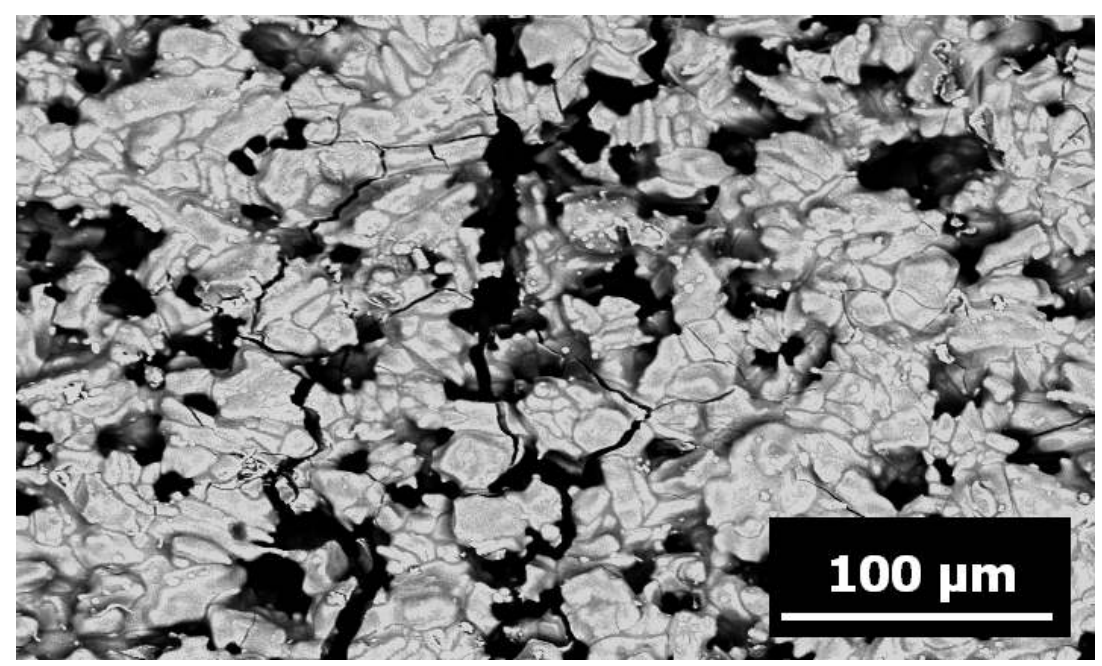

Fig. 2 SEM pictures (Back-Scattered Electrons) of the Fracture Process Zone for the aluminium titanate at room temperature - The main crack is coming from the bottom of the image 


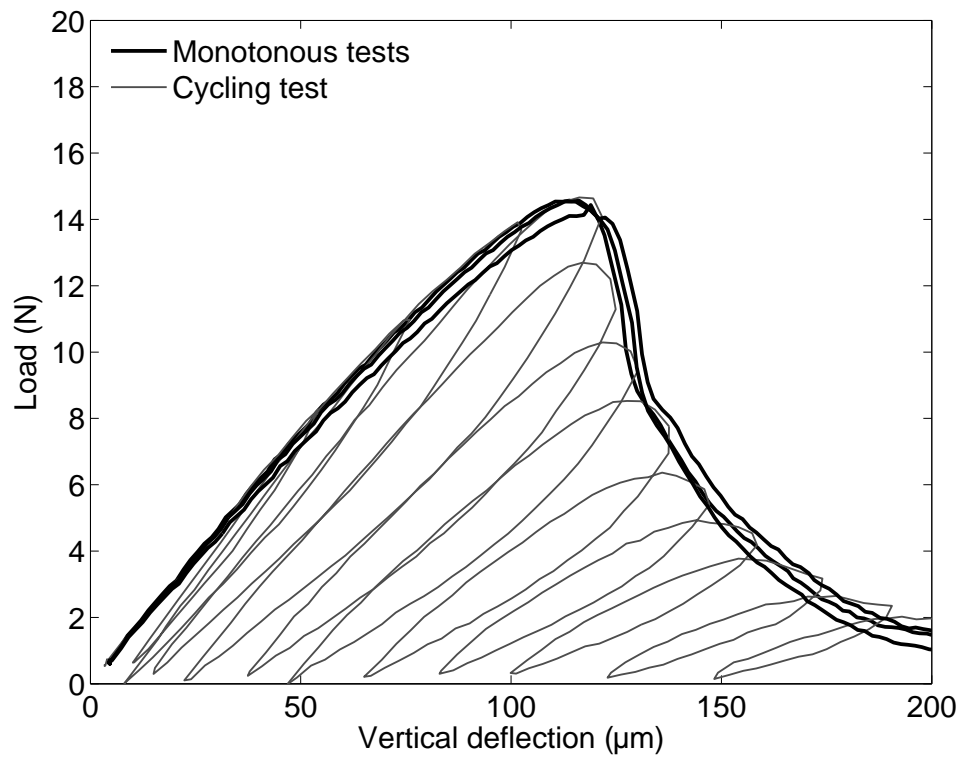

Fig. 3 Load-deflection curves for notched bending tests 


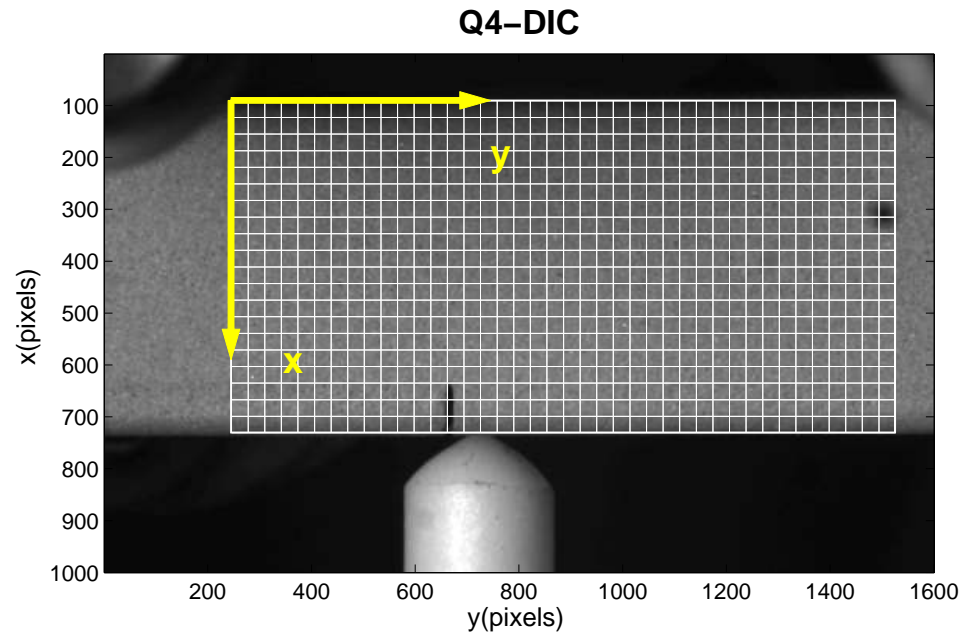

(a)

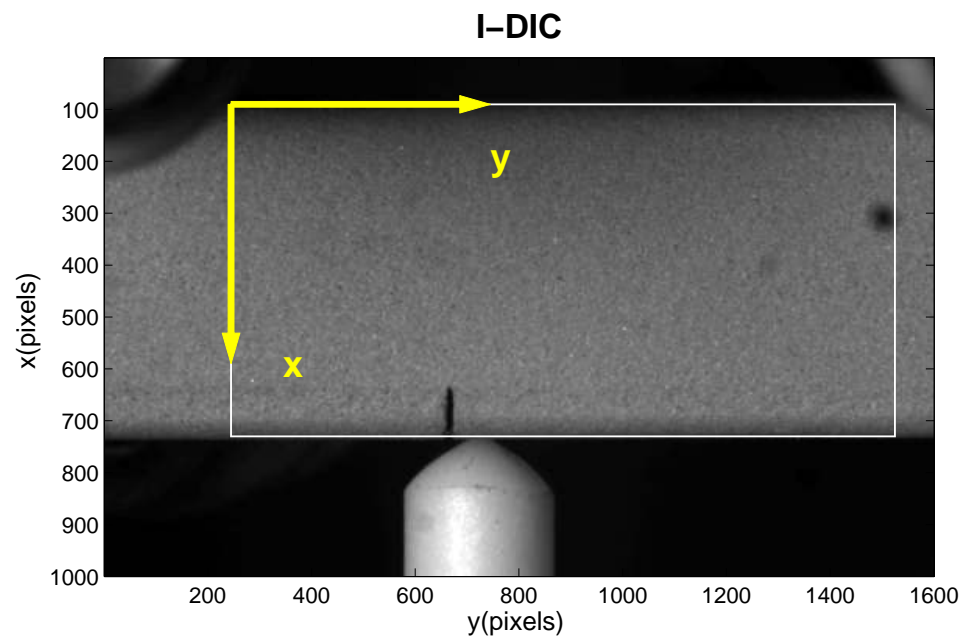

(b)

Fig. 4 Reference image and zones of interest for notched bending tests with FE-DIC (Q4 elements)(a) and I-DIC (b) - Pixel physical size $=10.9 \mu \mathrm{m}$ 

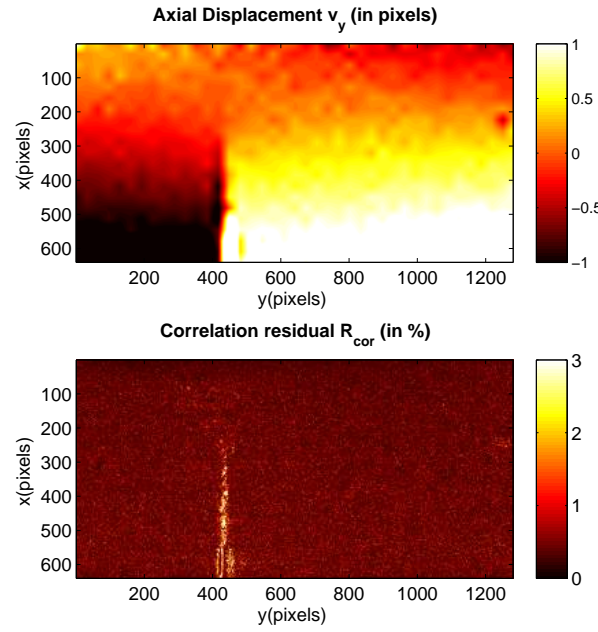

(a)
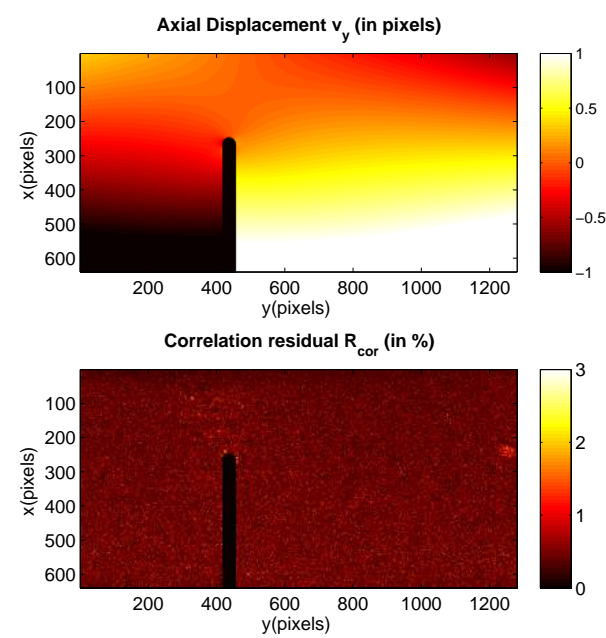

(b)

Fig. 5 Axial displacement $\left(v_{y}\right)$ and correlation residual $\left(R_{c o r}\right)$ maps. FE-DIC with standard Q4 interpolation (a) and I-DIC with Williams basis functions (b) 


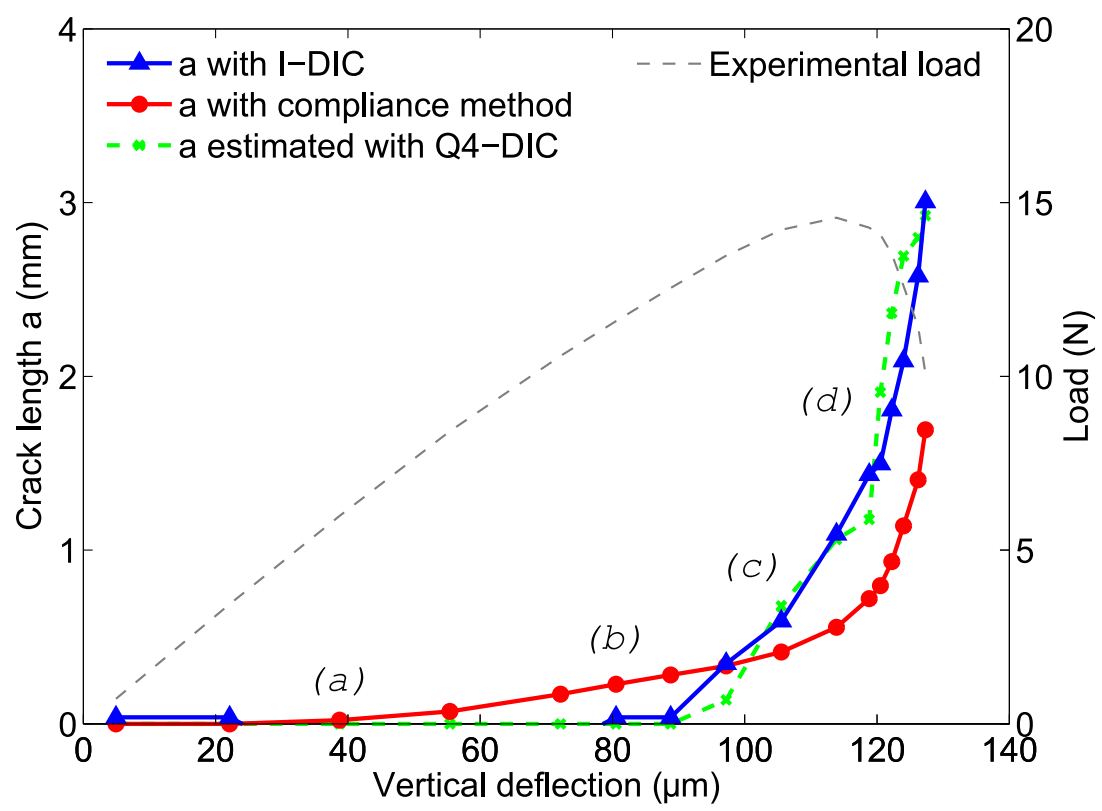

Fig. 6 Crack lengths a obtained using the compliance method and I-DIC. The letters corresponds to the load steps latter detailed in Figures (9) and (10)

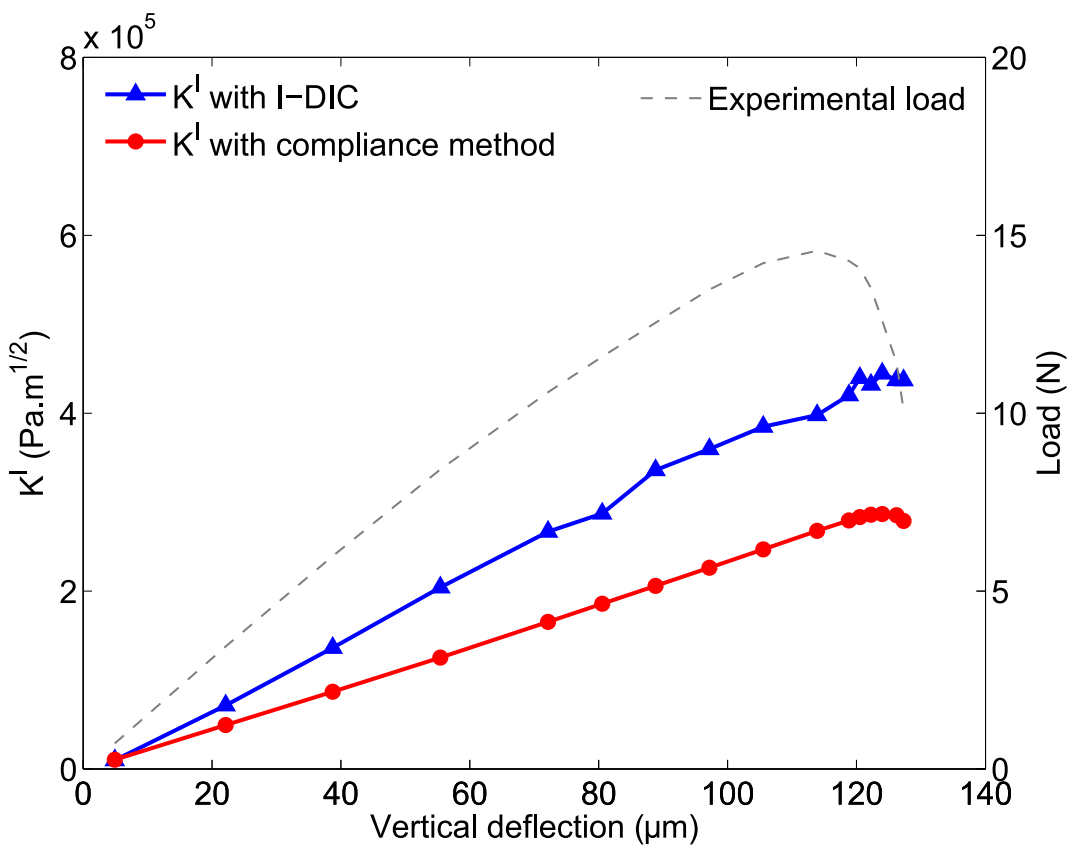

Fig. 7 Stress intensity factors $K^{I}$ obtained using the compliance method and I-DIC 


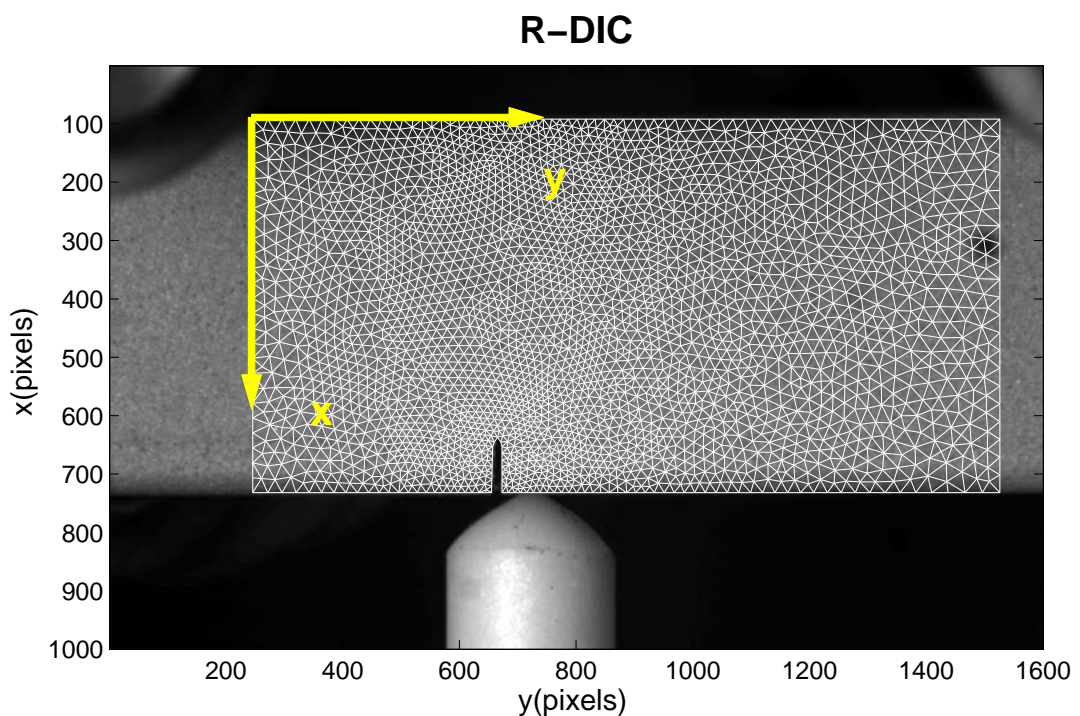

Fig. 8 Global zone of interest for notched bending tests using $R$-DIC - Pixel physical size $=10.9 \mu \mathrm{m}$ 

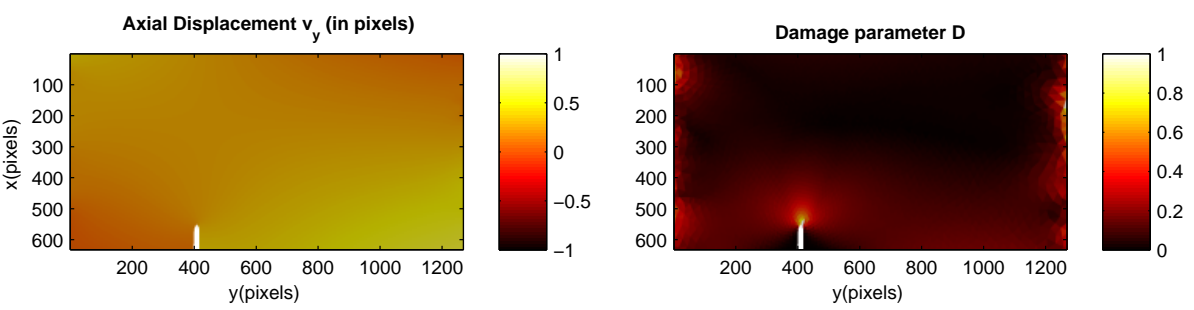

(a)
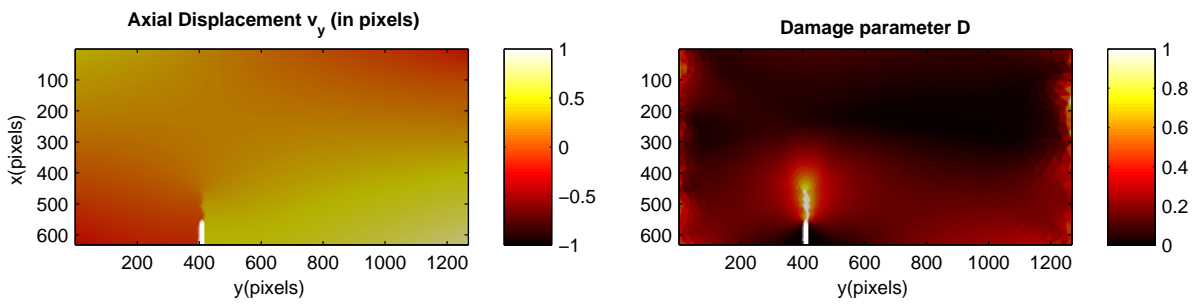

(b)
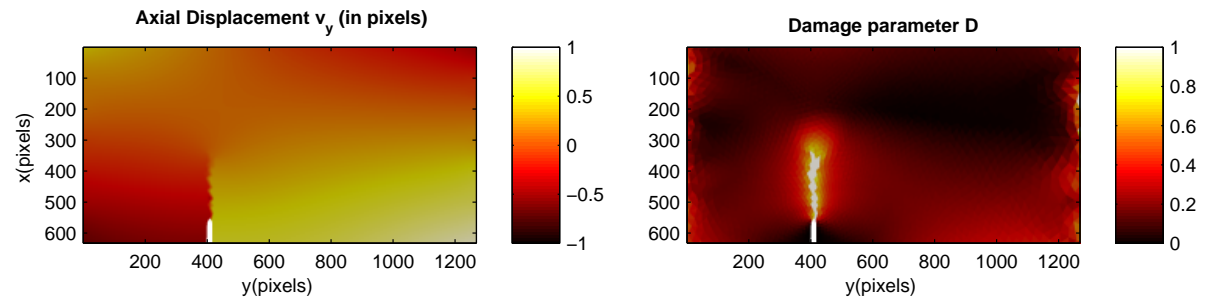

(c)
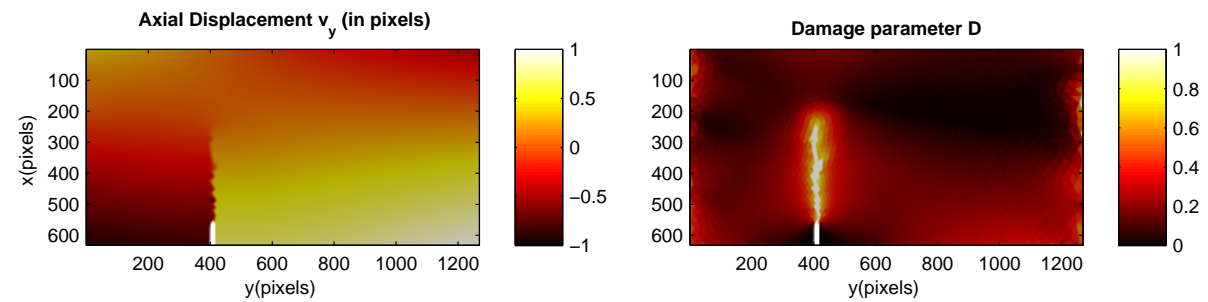

(d)

Fig. 9 Axial displacement $\left(v_{y}\right)$ and damage parameter $(D)$ obtained by $R$-DIC $\left(\lambda=4 \cdot 10^{-2}\right)$ for the four load steps labeled in Figure 6 


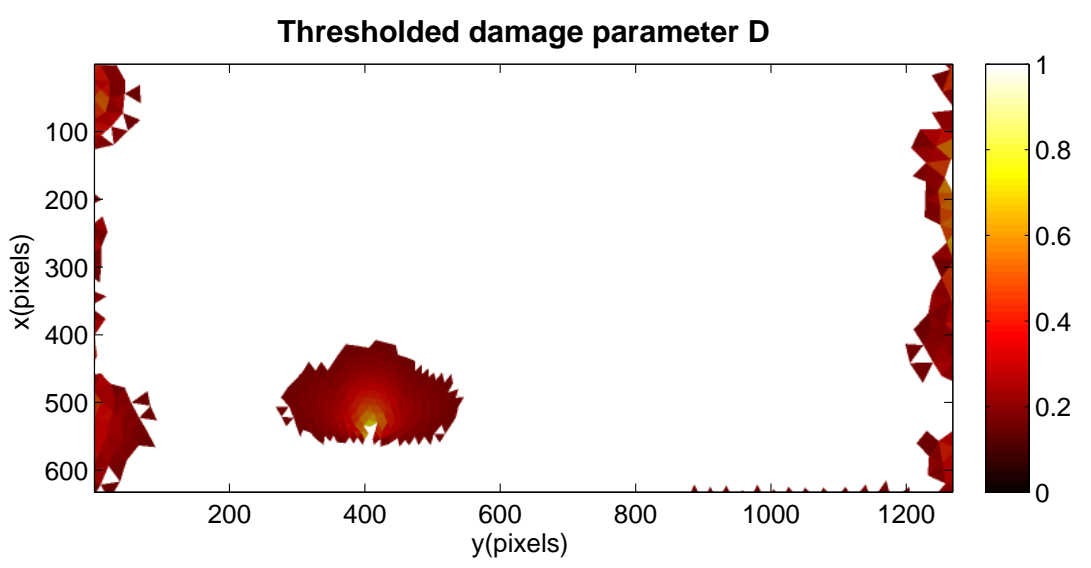

(a)

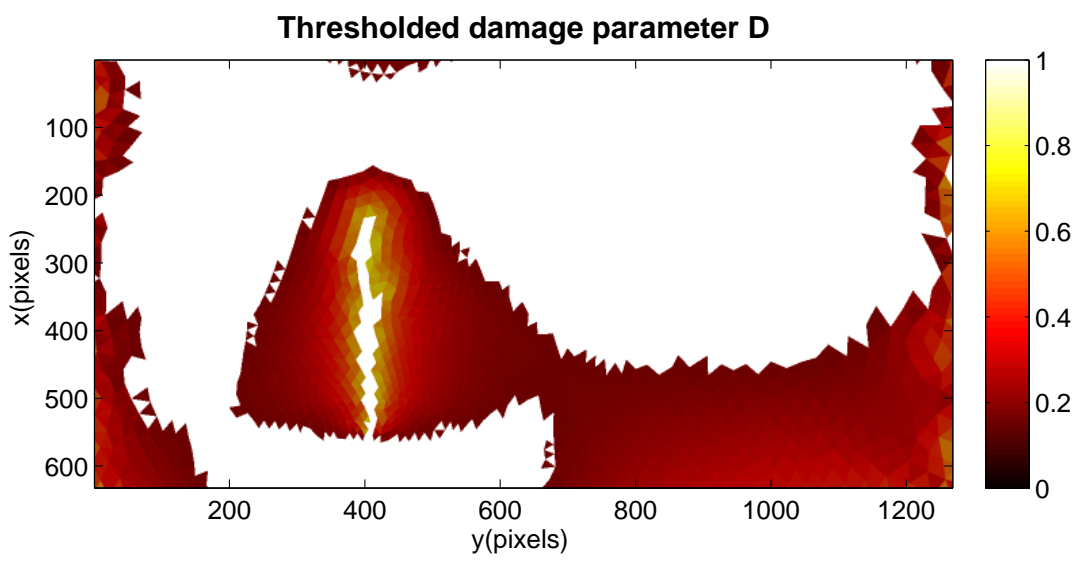

(d)

Fig. 10 Thresholded damage parameter $(D>0.2)$ obtained by $R$-DIC $\left(\lambda=4.10^{-2}\right)$ for the first and the last load steps described by Figure 9 


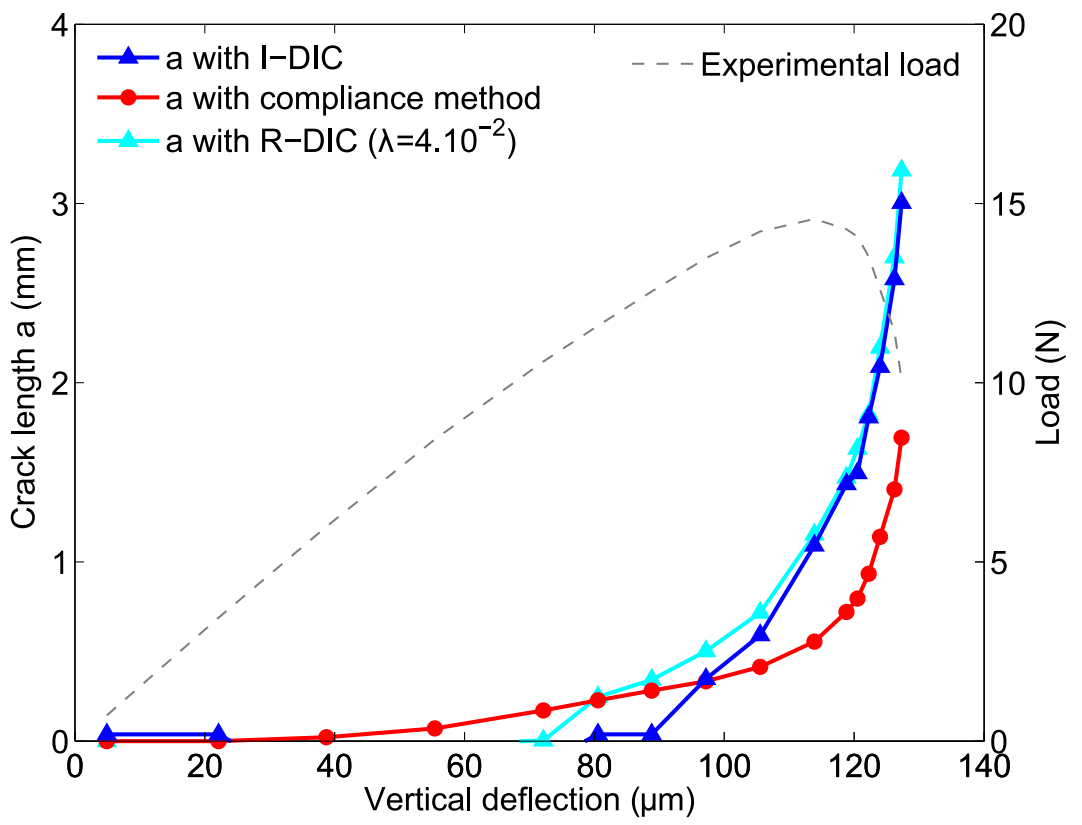

Fig. 11 Comparison of the crack length evolution from compliance, I-DIC and R-DIC methods

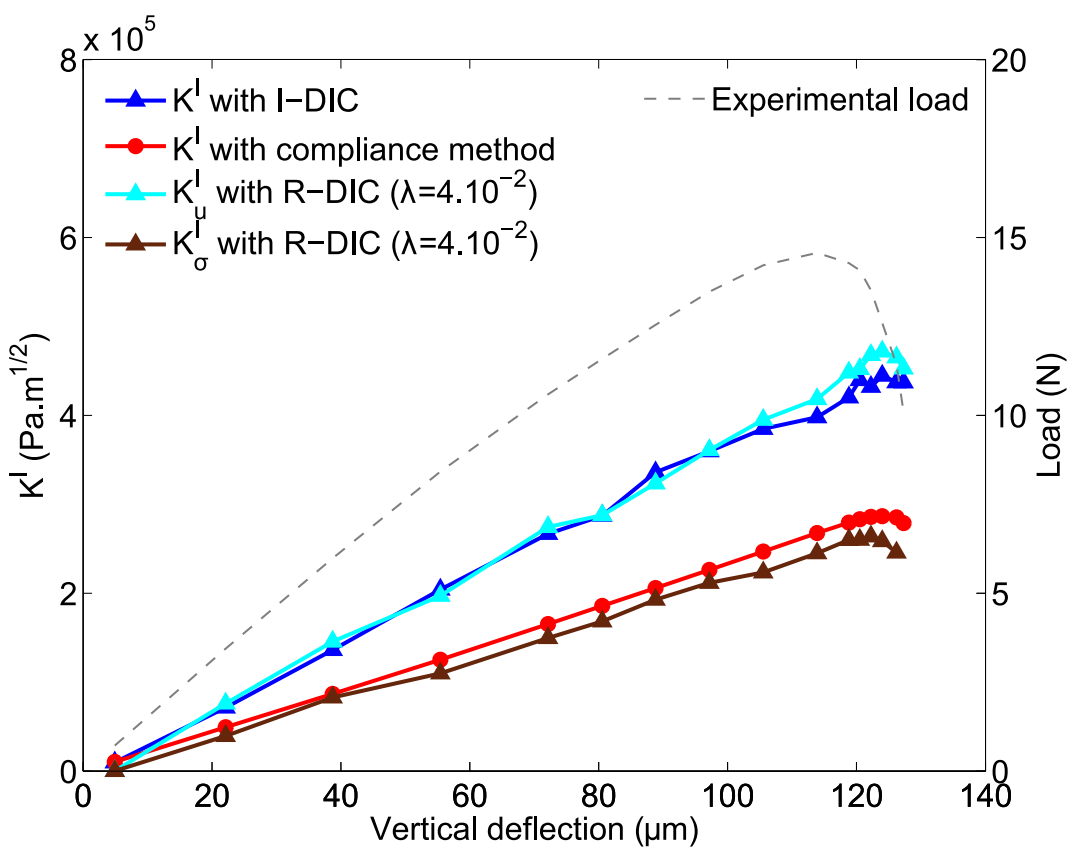

Fig. 12 Stress intensity factors $K^{I}$ obtained using the compliance method, I-DIC and R-DIC methods. For $R$-DIC both kinematic $K_{u}^{I}$ and static $K_{\sigma}^{I}$ stress intensity factors are plotted 


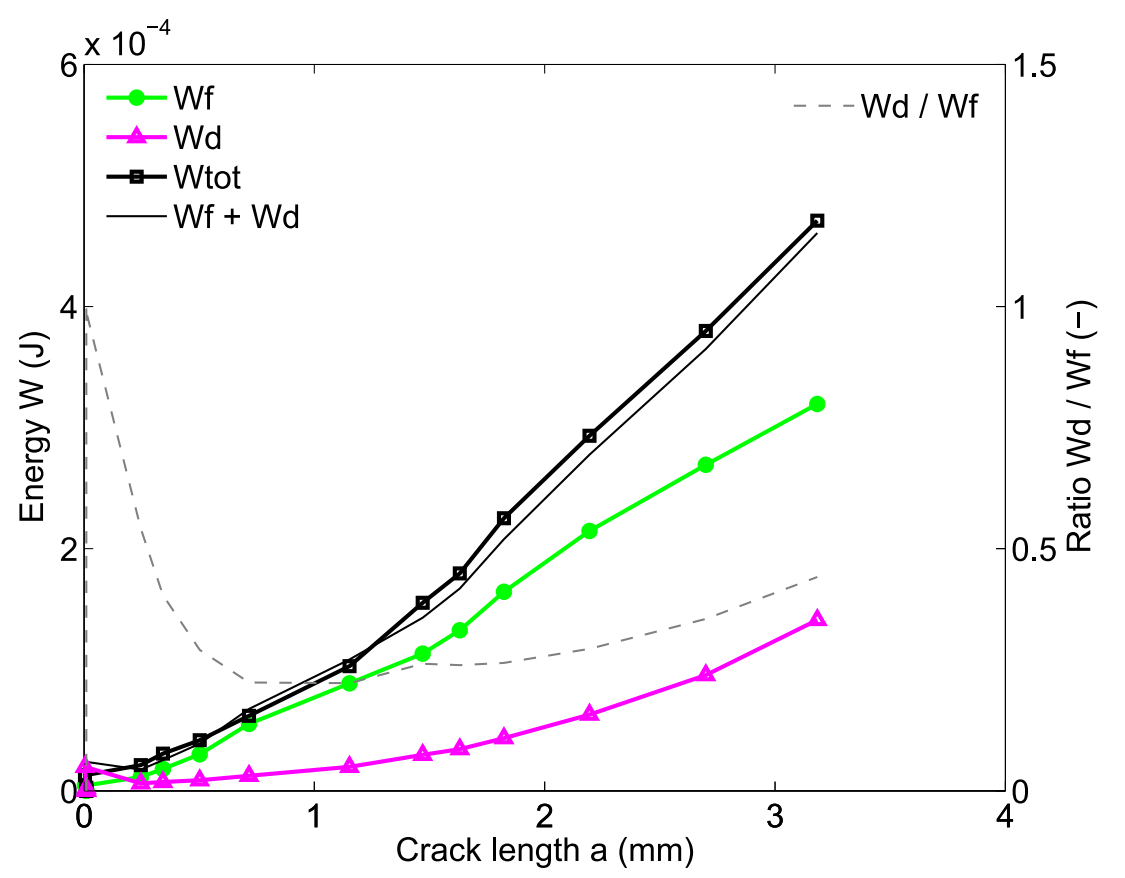

Fig. 13 Evolution of dissipated energy: total dissipation $W_{\text {tot }}$, dissipation by diffuse damage $W_{d}$ and dissipation by fracture $W_{f}$ 


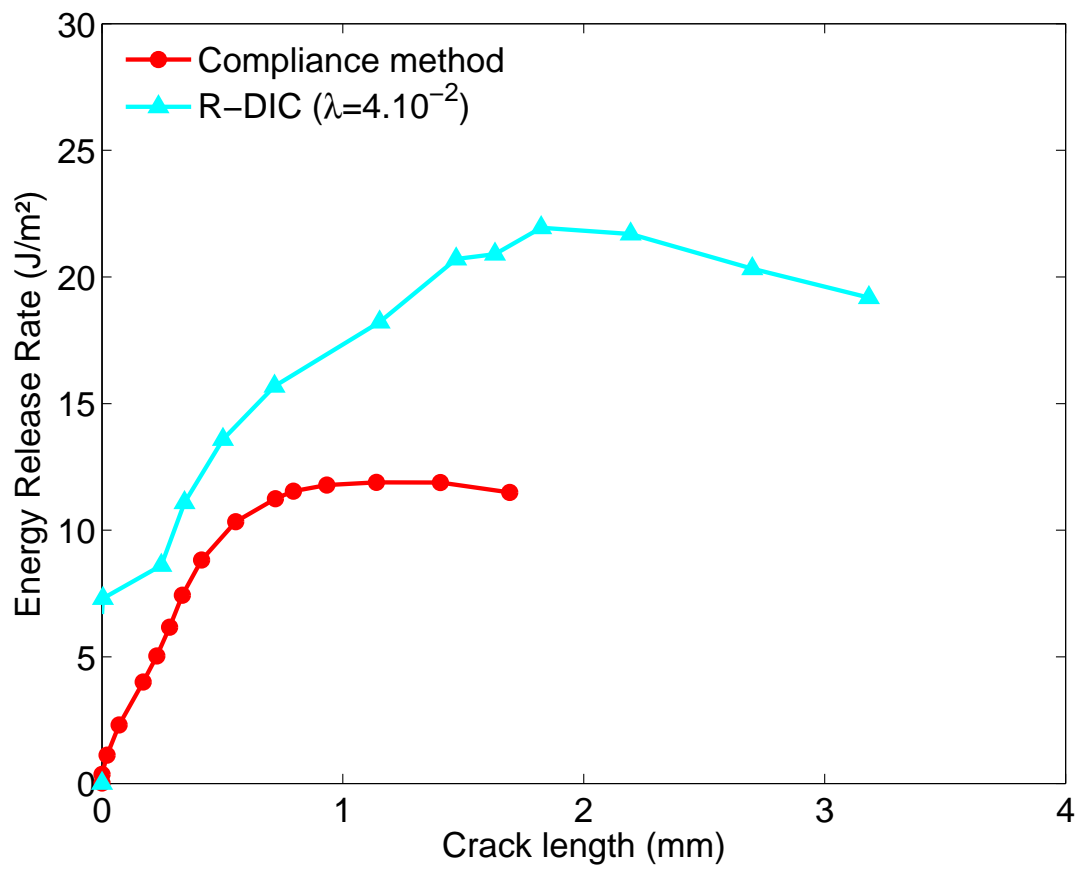

Fig. $14 R$-curves obtained according to compliance and $R$-DIC 